\title{
NUMERICAL MODELLING OF WIDE-SENSE STATIONARY GAUSSIAN RANDOM LOAD TORQUE SIGNAL
}

\author{
Guoxiang $\mathrm{Cao}^{1}$, Anlin Wang ${ }^{2}$, Donghuan $\mathrm{Xu}^{3}$ \\ seanxiang2012@tongji.edu.cn, wanganlin@tongji.edu.cn, \\ acelycol@tongji.edu.cn
}

\begin{abstract}
Dynamic load can reproduce the workload more realistically. It has great significance to the results of engineering machinery's simulation and bench test, but its parametric description and reproduction is difficult. this paper proposes a parameterized load model of Wide-Sense Stationary random gaussian signal to express and reproduce the signal. Dynamic loads can be decomposed into trend term and random term, and its dynamic characteristics are mainly concentrated in random term. In this paper, the method of white noise through linear system and impulse response method is used to reproduce the Wide-Sense Stationary gaussian random signal at automatic transmission output end. First, the data is fitted by polynomial, and the difference between the original data and the fitted curve as the trend term is taken as the random term. Then, after the ADF test is used to prove that the random term is Wide-Sense Stationary and obeys the gaussian distribution, the first-order linear system filter is obtained by fitting the power spectrum density of the random term. Finally, the reproduced Gaussian random term signal, whose power spectral density and statistical parameters are consistent with the original random term signal, is reproduced using the impulse response method.
\end{abstract}

Keywords: Wheel loader, Wide-Sense Stationary, Gaussian Random Signal, Power Spectrum Density.

\section{Introduction}

With the development of testing and simulation technology, in order to reduce the research and development cost and improve the test repeatability, the dynamic performance test research on the engineering machinery parts such as wheel loader is mostly carried out in the bench test or computer simulation environment. What kind of load signal is used has a great influence on the research result of the bench test or computer simulation environment.

If only simple signals such as constant load signal, step load signal, and slope load signal are used, which has great difference from the actual work load signal, it is bound to cause deviations in the conclusions of the study. If dynamic load signals are used, the workload can be reproduced realistically in bench test or computer simulation environment and the reliability of the research results can be improved.

However, the study on parametric model expression of wide-sense stationary random loads is relatively rare. Fu [1] studies the pressure dynamic expression at the output port of the hydraulic excavator multi-way valve, basically reproduces the dynamic load. He considers that the load is stationary in the cycle work conditions, but he has no proof that the load signal is widesense stationary. Wang [2-3] also doesn't prove the random load signal is widesense stationary, and he doesn't pay attention to the consistency of power spectrum density (PSD) between test load and reproduced load.

The dynamic load signal can be decomposed into trend term and random term, and the dynamic information of the load is mainly contained in the random term [4], however, its expression is difficult. To solve this problem, this paper proposes a parameterized load model of Wide-Sense Stationary random signal to express and reproduce the signal.

The transmission output shaft load torque signal of a wheel loader driving at a constant speed is selected as an example. First, the trend term is obtained by fitting the data with polynomial. The difference between the original data and the polynomial is the random term.

Then, the singular values of the random term signal are removed. After proving that the random term is Wide-Sense Stationary and obeys gaussian distribution, random term signal can be reproduced by white noise passing through a linear system which is determined by the PSD distribution of original signal.

Finally, the reproducing signal, whose statistical characteristics and the PSD distribution are consistent with the original signal, is obtained. Figure 1 is the flow chart of reproducing Wide-Sense Stationary gaussian random signal. 


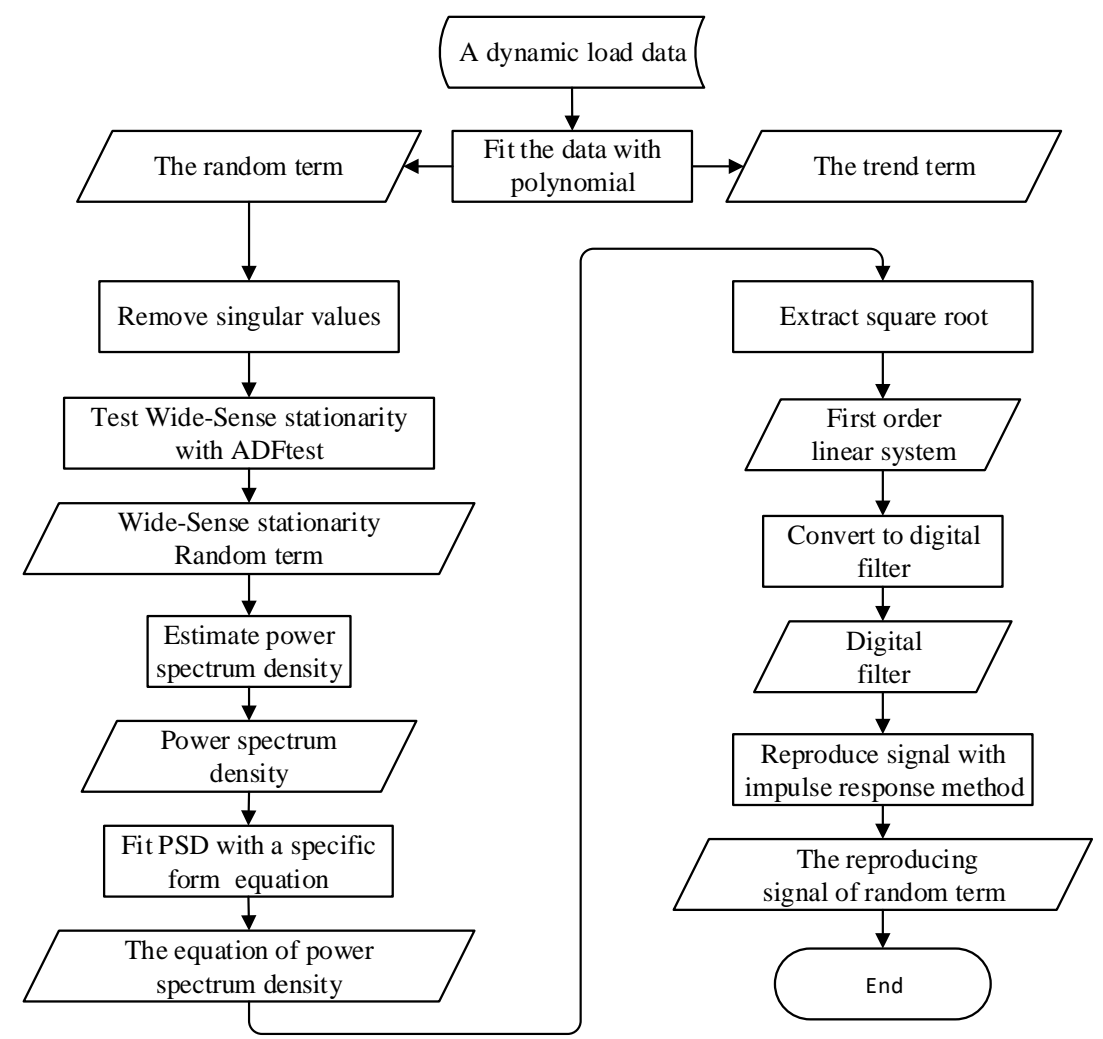

Figure 1. The flow chart of reproducing Wide-Sense Stationary gaussian random signal

\section{Characteristics of the Work Loads of Engineering Vehicles}

Construction machinery represented by loaders faces a harsh working environment. They must travel frequently on the roads such as the dirt road, the gravel road and other road surfaces. The road surface will exert a resistance torque on the tire. Since the road surface is uneven on the microscopic surface and the unevenness is large, the resistance torque acted on the tire is a random time series with a large variation range and a sharp change. These random loads can cause fatigue in parts and reduce component life. The dynamic random load also affects the shift quality of the automatic shifting process.

Dynamic random loads have different PSD distribution characteristics and statistical distribution characteristics due to differences in road conditions and the weight of the loader itself. Experiments test on wheel loader can provide real working conditions and dynamic load conditions, but a large number of experiments on loader are timeconsuming and labor-intensive, which is unacceptable to the enterprise. Now, thanks to the development of simulation technology, computer simulation can be used to model the wheel loader system, improve the efficiency of research and development, and save time and money.
Therefore, it is necessary to study the parametric expression and simulation of dynamic random loads.

The torque comes from load. In order to more realistically study the performance of the whole machine and components, it is necessary to accurately reproduce the experimental load torque characteristics of the whole machine test. And the dynamic random load is mainly described by PSD distribution characteristics and statistical characteristics. Therefore, the PSD distribution characteristics and statistical characteristics of the dynamic random load must be paid attention to ensure them consistent with the load measured in wheel loader test experiment.

\section{Pre-Processing of Load Signal}

\subsection{Decomposition of load signal into trend term and random term}

The experimental platform used in this paper is the ZL50 wheel loader. A typical work condition is selected. The wheel loader drives straightly at a constant speed on the flat slag land in the state of full load, 1st gear and fully accelerator pedal. The torque sensor is placed on the output shaft of the gearbox in figure 2 and the sampling frequency is $10 \mathrm{~Hz}$.

Load can be decomposed into the trend term and the random term. The load data of $25.6 \mathrm{~s}$ is selected. 
The data is fitted with a polynomial of 7 times. The original data and polynomial curves are shown in the figure 3 . The difference between the original data and the fitting data is taken as the random term.

The random term data is shown in the figure 4.

The dynamic information of load is mainly included in the random term, and the following content is mainly focused on the analysis and processing of random term.

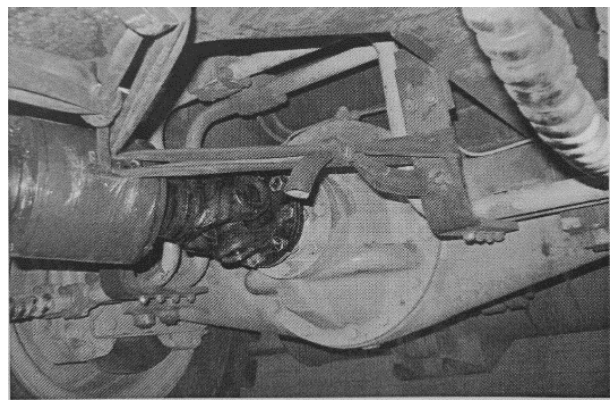

Figure 2. Test on torque measurement of transmission shaft

Singular values may occur in the measurement process due to electromagnetic interference or sensor vibration. This paper uses the standard deviation detection method to remove singular values in the random term that exceed three times the standard deviation.

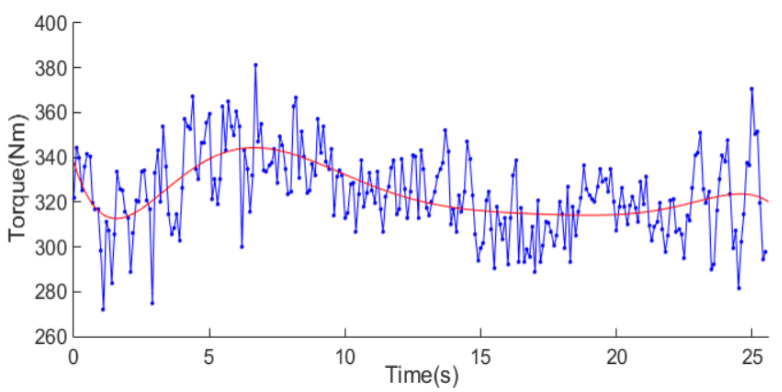

Figure 3. The load data and polynomial fitting curve.

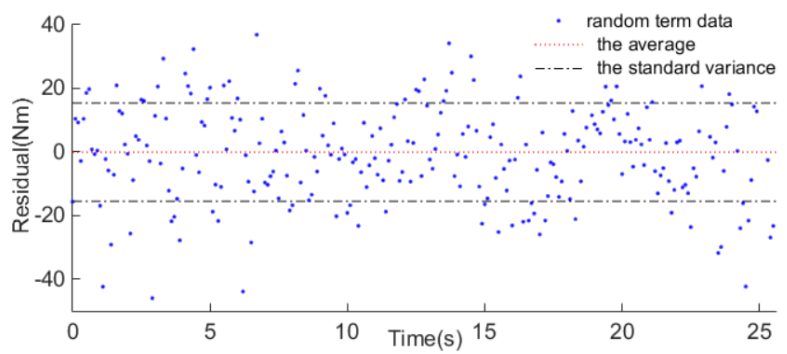

Figure 4. The residual, average and standard variance

\subsection{Proving of wide-sense stationarity of random term signal}

If all n-order probability density distribution of a random signal $\boldsymbol{X}$ (is independent of the time start, and does not change with time, $\quad \boldsymbol{X}$ ( is Strict Sense Stationarity signal.

$$
\begin{aligned}
& f_{x}\left(x_{1}, x_{2, \ldots \ldots} x_{n,}, t_{1}, t_{2, \ldots} t_{n}\right)=
\end{aligned}
$$

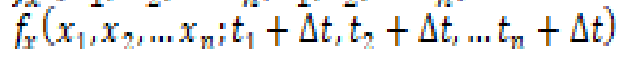

Since a Strict Sense Stationarity signal requires all n-order probability density distribution stationary, it is very difficult to determine that all norder probability density distribution stationary of a signal is stationary in practice. On the other hand, in engineering practice, people tend to pay more attention to the first and second moments of random signals. So, there is a definition of a Wide-Sense Stationary signal. If the first and second moments of the signal is independent of the time start, and does not change with time, it is a Wide-Sense Stationary signal.

Augmented Dickey-Fuller (ADF) [5] test is a common method for checking the Wide-Sense stationarity of time series. ADF test is an important single root detection method. The initial hypothesis is that the sequence is un-stationary, and the detection can be performed in two ways. The first method is that if the 'Test Statistic' is less than the 'Critical Value', we can reject the null hypothesis and say that the series is stationary. The second is that If $\mathrm{p}$-value is above a critical size, then we cannot reject that there is a unit root.

The ADF method is applied to test the random term, and return p-value 0.001 , so we can reject the assumption that there is a unit root. In other words, the sequence is stationary. Only random term is stationary, then the stochastic signal can be processed by the relevant methods of stationary stochastic theory.

\subsection{Gaussian distribution of random term signals}

The Jarque-Bera test (jbtest) is a method to determine whether the sample data have the skewness and kurtosis matching a normal distribution. The jbtest result of the random term signal is that it obeys normal distribution. And the result can be visualized in Figure 5. If the data are normal the plot will be linear. Obviously, the data is normal distribution.

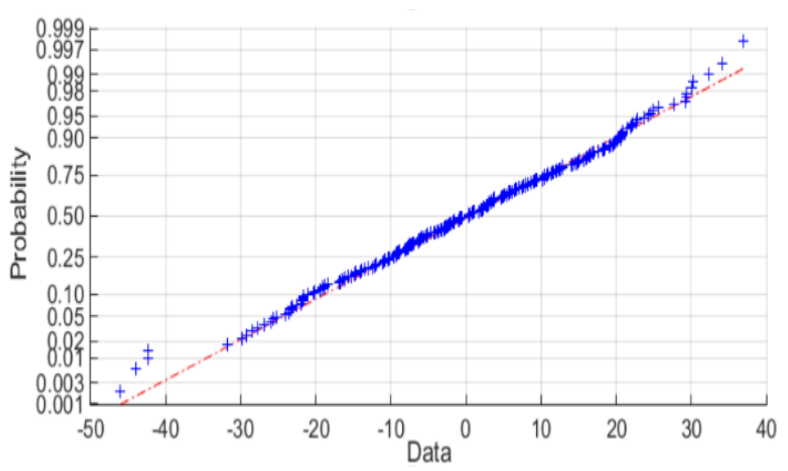

Figure 5. The normal probability plot 


\section{Parameterized Model of Random Term Load Signal}

\subsection{Power spectrum density estimation of random term load signal}

The Welch method is used to estimate the power spectrum of the random load term, and the obtained PSD curve is shown in figure 6. It can be seen that the PSD of the random load is limited, the energy is basically distributed in $0 \sim 2 \mathrm{~Hz}$, and the trend is gradually decreasing.

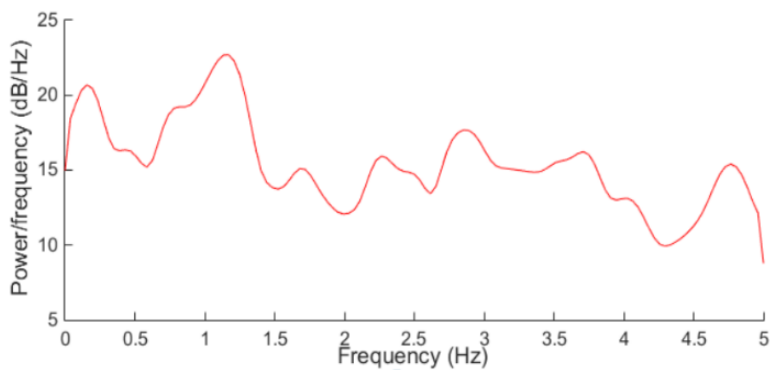

Figure 6. The PSD of random term by Welch method

\subsection{Parameterized model of random term load signal}

The PSD of white noise is flat and infinitely wide.

When the white noise with a PSD of $N_{0 \text {. }}$ passes through a low-pass system with a transfer function of $\mathrm{H}$ (i, the PSD of the output signal is

$$
s_{y}(\omega)=\frac{N_{0}}{2}|\mathrm{H}(\omega)|^{2}
$$

And the output signal obeys a Gaussian distribution. According to the form of the PSD curve,

The PSD of the random term can be fitted in the form of equation (3).

$$
S_{y}=\frac{K}{1+(a * 2 \pi f)^{2}}
$$

where and are the parameter to be determined. If a white noise with a PSD of $1 / 2$ passes through the linear system equation (4),

$$
\mathrm{H}(\mathrm{s})=\frac{\sqrt{K}}{1+a * s}
$$

the PSD of output signal can be consistent with the curve of equation (3). Before the digital simulation with method of the impulse response, the analog filter is first converted to a digital filter.

$$
\mathrm{H}(\mathrm{z})=\frac{\sqrt{K}}{1-e^{\frac{-a}{f s}} z^{-1}}
$$

Finally, the parameterized model of random term load signal is got and the reproduced signal consistent with the PSD of the original random term can be obtained [6].

\section{The Results of Reproducing Random Term Signals}

The parameterized model equation (5) can reproduce a regenerative random signal in MATLAB.

The PSD distributions and fitting curves of the random term signal and the reproducing signal are showed in the figure 7 . It can be concluded from the results that the two signals' PSD distributions and fitting curves are almost the same.

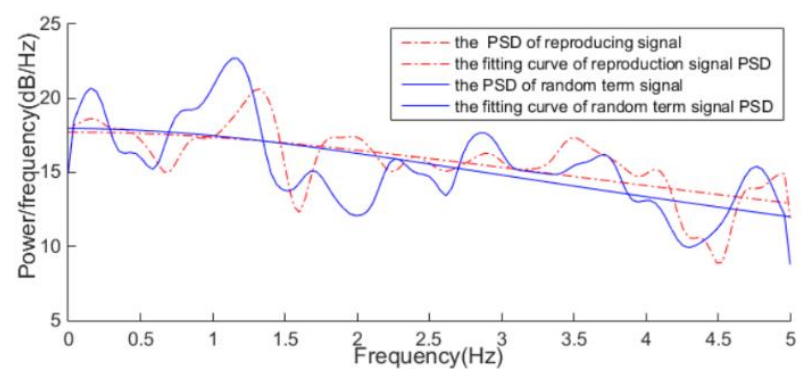

Figure 7. The PSD and fitting curve of reproducing signal and random term signal

The figure 8 is the histograms and fittings of two signals, whose histogram distributions are obviously basically the same. And they all obey the normal distribution according to the normal distribution probability fitting in figure 9. Besides, the statistical parameters of the original random term signal and the regenerated random signal are compared in table 1.

We can find that the related statistical parameters such as the average, the standard deviation, of the original signal and the regenerative signal are basically consistent. Therefore, using the impulse response method to simulate the random term signal not only ensures the consistency of the PSD, but also ensures the statistical parameters nearly equal. In other words, the method of white noise through a linear system can reproduce the original signal more realistically.

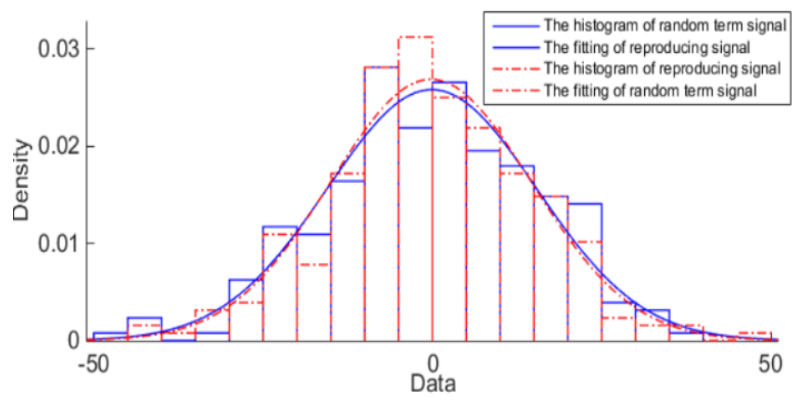

Figure 8. The histograms and fittings of two signals 


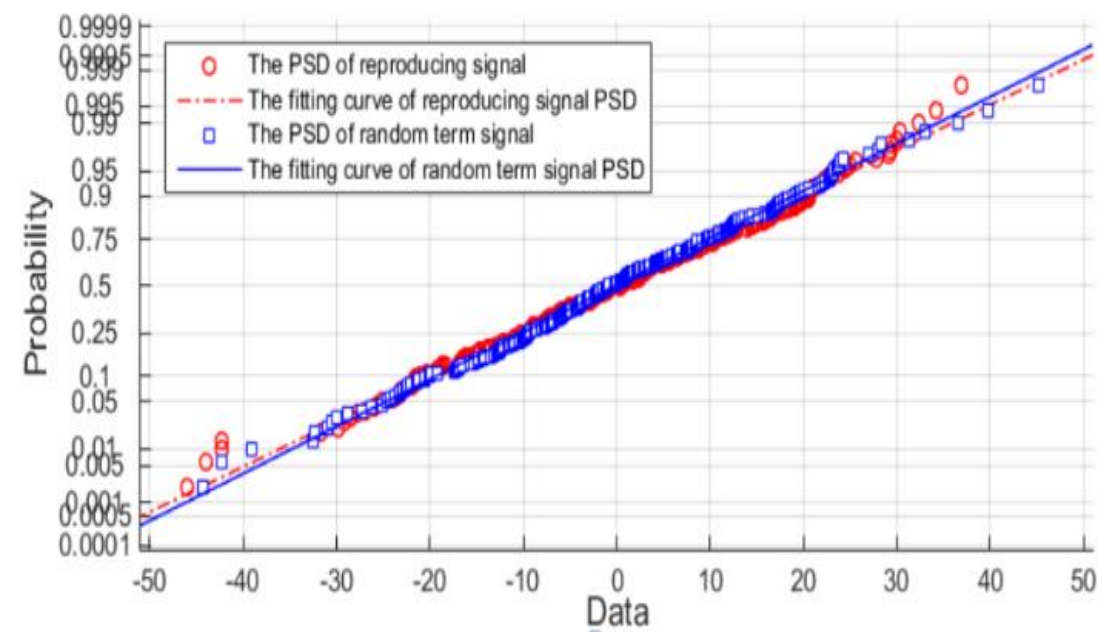

Figure 9. The normal distribution probability of two signals

Table 1. Statistical parameters of the original random term signal and the regenerated random signal

\begin{tabular}{lllll}
\hline Random term & Maximum & Minimum & Average & Standard deviation \\
\hline The original & 36.88 & -46.02 & -0.0714 & 15.45 \\
The reproduced & 43.08 & -40.7 & -1.008 & 14.57 \\
\hline
\end{tabular}

\section{Discussion and Conclusion}

From the perspective of wide-sense stationarity random signals, this paper prove that the random term load signal is Wide-Sense Stationary and obeys Gaussian distribution. The random term signal is reproduced by the method of the white noise passing through a linear system. This method can ensure that the PSD and the statistical parameters of the reproduced signal and the original signal are consistent.

The parametric model of the random term signal is established by using a typical working condition data, which can reproduce a random signal consistent with the original signal. This means may be applied in simulation test and bench test to simulate the load random term signal to improve the reliability of the research results.

For example, a synthetic load signal of a constant torque signal and a dynamic torque regeneration signal can be used in the experiment to generate a specific working condition. It can reproduce the working environment of the experimental objects more realistically and improve the reliability of the experimental results.

\section{Acknowledgments}

We are grateful for the research grants from the Ministry of Industry and Information Technology
National Major Scientific and Technological Achievements Transformation Project.

\section{References}

[1] J. Tao, Z. FU, and A. WANG. "Parameterized Load Model Under Non-Stationary Random Cyclic Conditions." Journal of Northeastern University (Natural Science) 6 (2016): 018.

[2] WANG, Jixin, et al. A cyclic simulation approach for the generation of the non-stationary load histories of engineering vehicles. Journal of mechanical science and technology, 2012, 26.5: 1547-1554.

[3] LIANG, Y. L. Compiling method of load spectra and signal reconstruction in time-domain for hydraulic cylinders of wheel loader. Master degree thesis: Jilin University, 2011.

[4] MA, Deng-cheng, et al. An analysis of fluctuations load and simulation method on construction machinery. Journal of Gansu Agricultural University, 2013, 3: 027.

[5] WORDEN, K.; IAKOVIDIS, I.; CROSS, E. J. On Stationarity and the Interpretation of the ADF Statistic. In: Dynamics of Civil Structures, Volume 2. Springer, Cham, 2019. p. 29-38.

[6] P. Luo, and W. Zhang, Random signal analysis and processing. Tsinghua university press co. LTD, (2006), P101. 